\title{
Be part of the chain
}

There is room for improvement in materials sustainability, provided we examine carefully the whole value chain.

W

hat can materials science do for the sustainable development of our planet? This has been a recurrent question in Nature Materials ${ }^{1-3}$. Well-defined paths have been traced to address the challenge, including the development of ever more efficient systems for the production, storage and distribution of clean energy, carbon capture and storage approaches, and strategies to reduce the environmental impact of materials in high demand such as concrete and metals ${ }^{3,4}$. But how should these approaches be implemented to reach the goals set by the Paris agreement on greenhouse gas (GHG) emissions ${ }^{1}$ ? What will be the impact on raw-materials demand? Are there additional paths that we could take to be more effective? To discuss these topics, in September 2018 materials scientists met with experts in Earth science, environmental engineering and economics in Trondheim, at the Nature Conference on Minerals and Materials for a Sustainable Future organized by the Geological Survey of Norway (NGU), the Norwegian University of Science and Technology, and SINTEF Industry, in collaboration with Nature Geoscience and Nature Materials.

Saleem Ali, professor at the University of Delaware, USA, and University of Queensland, Australia, discussed at the conference - and also in a correspondence in the issue - his views as a member of the Global Future Council on Advanced Materials of the World Economic Forum. Reducing GHG emissions is one of the three key areas identified by the council where advanced materials can have an impact. Technological innovations are certainly needed to achieve this goal; yet their deployment at scale requires minerals and other natural resources that must be taken into account in the overall equation. As such, we need to look at the whole materials value chain to identify ways to improve resource efficiency.

The consensus is that, at least in the short term, there will be no shortage of raw materials to fabricate solar panels, wind turbines or units for electricity storage. Yet a thorough analysis will be required to understand if a faster pace of transition to renewable energy sources - deemed necessary to limit global warming to $1.5^{\circ} \mathrm{C}$ above pre-industrial levels ${ }^{5}$ - may make resource availability an issue. Importantly, the metal content of some ores is decreasing, meaning that more energy will be required to extract and process raw materials further contributing to climate change ${ }^{6}$.

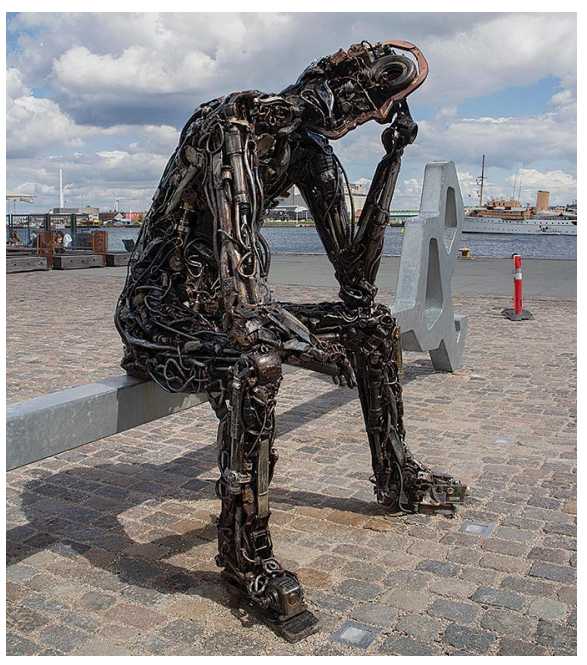

The Global Visionary by Kim Michael. Credit: Peter K. Lloyd/Alamy Stock Photo.

Specific investments are needed to produce detailed geological maps and identify new sites for minerals extraction; yet new mines will hardly be fully operational in less than ten years, according to Tom Heldal, director of the Division for Geological Resources and Environment at NGU.

Resorting to secondary material resources through end-of-use product recycling is also a widely explored strategy. Unfortunately, for many elements this recycling process is far from ideal ${ }^{7}$ : more than $50 \%$ of the material flows are lost due to in-use dissipation or because current recycling technologies are unable to separate and recover the individual elements. Furthermore, the properties of a large fraction of the potentially recoverable materials are irreversibly downgraded. Karen Hanghøj, chief executive officer at EIT RawMaterials, sees plenty of space for improvement here: by taking materials recovery into account at earlier stages in the value chain, goods could be designed to facilitate disassembly and materials separation at end of use. Yet she also warns that, for materials with rising demand (such as minerals and metals used in photovoltaics and other green technologies), there will inevitably be a need for the supply from primary sources. Simplification of chemical composition would also make recycling easier - Professor Alexander King from Iowa State University highlighted that it was simpler to recover gold from the first mobile phones, which used only 30 elements, with respect to current smart phones produced with about 65 different elements. Reduced complexity would also make companies less vulnerable to materials criticalities due to geopolitical reasons, certainly an added value for interested stakeholders.

However, more has to be done. According to Julian Allwood, professor at the University of Cambridge, there is a significant danger that optimism about future technological innovations is preventing us from taking actions to reduce our overall demand for materials. The deployment of new technologies to produce clean energy, electrify the industrial sector and capture carbon emissions is unlikely to reach the scale and speed needed to limit global warming to $1.5^{\circ} \mathrm{C}$, if not supported by complementary actions. In another correspondence in this issue, Allwood warns against entrusting new technologies without assessing their scalability, and suggests additional strategies to decrease demand for resources and use them more efficiently. Adjustments in the design of goods, from cars to buildings, may reduce the amount of primary materials scrapped during manufacturing and those used in the products themselves ${ }^{8}$. Smaller and lighter cars can still accomplish their primary transport functions while saving materials and reducing fuel consumption. Finally, extending the lifespan of material-intensive products would decrease the need for new materials manufacturing.

Clearly there are plenty of opportunities for materials scientists and engineers to make an impact on the sustainable development of the world. In order to identify the most effective steps, however, we need to communicate with geologists, environmentalists, economists and social scientists to avoid compartmentalized views and gaps in understanding the scale of this challenge.

Published online: 23 November 2018 https://doi.org/10.1038/s41563-018-0245-8

References

1. Nat. Mater. 15, 113 (2016).

2. Materials for sustainable energy. Nat. Mater. https://www.nature. com/collections/pwybcffhb (2017).

3. Sustainable materials. Nat. Mater. https://www.nature.com/ collections/gfvhbtjwss (2017).

4. Chu, S., Cui, Y. \& Liu, N. Nat. Mater. 16, 16-22 (2017).

5. Special Report on Global Warming of $1.5^{\circ} \mathrm{C}$ (IPCC, 2018); http:// www.ipcc.ch/report/sr15/

6. Raw materials use to double by 2060 with severe environmental consequences. OECD https://go.nature.com/2FdBfOT (2018).

7. Ciacci, L., Reck, B. K., Nassar, N. T. \& Gradel, T. E. Environ. Sci. Technol. 49, 9443-9451 (2015).

8. Allwood, J. M. \& Cullen, J. M. Sustainable Materials: Without the Hot Air (UIT Cambridge, Cambridge, 2015). 